\title{
On difunctionality of class relations
}

M. Hoefnagel]Michael HoefnagelDepartment of Mathematical Sciences Stellenbosch University Private Bag X1 Matieland 7602 South Africa

Z. Janelidze]Zurab Janelidze Department of Mathematical Sciences Stellenbosch University Private Bag X1 Matieland 7602 South Africa Second author's research is partially supported by the South African National Research Foundation

D. Rodelo]Diana Rodelo Departamento de Matemática Faculdade de Ciências e Tecnologia Universidade do Algarve Campus de Gambelas 8005-139 Faro Portugal and CMUC, Department of Mathematics University of Coimbra 3001-501 Coimbra Portugal The third author acknowledges partial financial assistance by the Centre for Mathematics of the University of Coimbra - UID/MAT/00324/2019, funded by the Portuguese Government through FCT/MEC and co-funded by the European Regional Development Fund through the Partnership Agreement PT2020.

keywords: Class relations, Congruence distributivity, Congruence modularity, Congruence permutability, Directly decomposable congruence classes, Difunctionality, Egg-box property, Mal'tsev condition, Mal'tsev variety, Shifting lemma

\begin{abstract}
For a given variety $\mathcal{V}$ of algebras, we define a class relation to be a binary relation $R \subseteq S^{2}$ which is of the form $R=S^{2} \cap K$ for some congruence class $K$ on $A^{2}$, where $A$ is an algebra in $\mathcal{V}$ such that $S \subseteq A$. In this paper we study the following property of $\mathcal{V}$ : every reflexive class relation is an equivalence relation. In particular, we obtain equivalent characterizations of this property analogous to well-known equivalent characterizations of congruence-permutable varieties. This property determines a Mal'tsev condition on the variety and in a suitable sense, it is a join of Chajda's egg-box property as well as Duda's direct decomposability of congruence classes.
\end{abstract}

\section{Introduction}

This paper originated with an observation of the third author that the shifting lemma [13] in a variety can be used to deduce that classes $K$ of congruences on a product $X \times U$ of two algebras are difunctional relations in the sense of [19], i.e., if $(x, u),(x, v)$ and $(y, v)$ are in the class, then so is $(y, u)$. Indeed, this can be deduced at once from the following particular instance of the diagram in the 
shifting lemma:

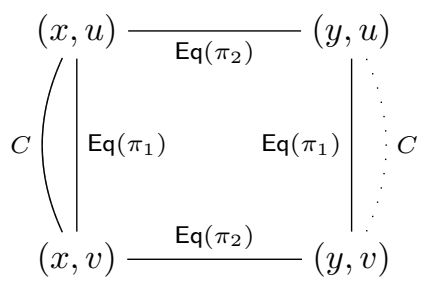

Recall from [16] that the property of difunctionality of homomorphic relations is equivalent to congruence-permutability, which implies congruence-modularity; the latter is in turn equivalent to the shifting lemma, as shown in [13]. Congruencemodularity is strictly weaker than congruence-permutability, and so the question of identifying difunctional relations in a congruence-modular variety is of some interest.

In this paper we study the property of difunctionality of relations $K$ as considered above, i.e., those relations which arise as congruences classes on a cartesian product of two algebras. Such relations are a particular type of 'class relations' defined in this paper, which are also difunctional when the $K$ 's are. In the main theorem of the paper (Theorem 1.3) we show that the well-known characterizations of congruence-permutable varieties in terms of properties of homomorphic relations carry over to the case when similar properties are considered for class relations. The challenge here was not in the proof of the theorem, which follows an argument similar to the one used in the case of congruencepermutability, but rather in its formulation, which shaped the present concept of a class relation. Among other things, in this theorem we establish that difunctionality of class relations (DCR) is equivalent to every reflexive class relation being an equivalence relation, as well as permutability $E_{1} E_{2}=E_{2} E_{1}$ for equivalence relations $E_{1}$ and $E_{2}$ such that $E_{1} E_{2}$ is a class relation. We also show that (DCR) is a Mal'tsev condition on a variety (Theorem 1.6).

The generalization of the shifting lemma given by the diagram condition above is precisely the egg-box property (EBP) in the sense of [3]. There is another similar diagram from which we can deduce (DCR):

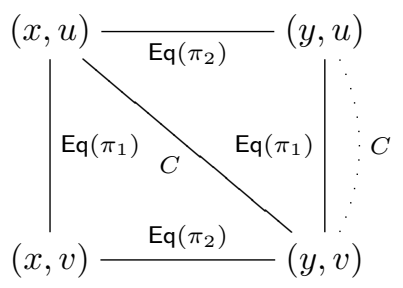

This diagram defines varieties with directly decomposable congruence classes (DDCC) in the sense of [8]. Thus (DCR) is a common consequence of (EBP) and (DDCC). This property is nevertheless restrictive enough not to hold, for instance, for certain subtractive varieties and certain Jónsson-Tarski varieties (Theorems 3.1 and 3.2). 
Expressing (EBP) as the formula

$$
(x, u) C(x, v) \Rightarrow(y, u) C(y, v)
$$

and (DDCC) as the formula

$$
(x, u) C(y, v) \Rightarrow(y, u) C(y, v),
$$

we find that the formula

$$
[(x, u) C(x, v) \wedge(x, u) C(y, v)] \Rightarrow(y, u) C(y, v)
$$

that expresses (DCR) is (after ignoring the hidden universal quantifiers) nothing but the disjunction of the previous two formulas, as in general,

$$
[\alpha \Rightarrow \beta] \vee\left[\alpha^{\prime} \Rightarrow \beta\right]
$$

is equivalent to

$$
\left[\alpha \wedge \alpha^{\prime}\right] \Rightarrow \beta .
$$

In this way, we can view (DCR) as a 'join' of (DDCC) and (EBP). On the other hand, taking the conjunction of first two formulas we get a formula expressing the conjunction of (DDCC) and (EBP), which was shown in [4] to be equivalent to direct decomposability of congruences (DDC). Thus, the property (DCR) that we study in this paper naturally fits into a diamond of properties:

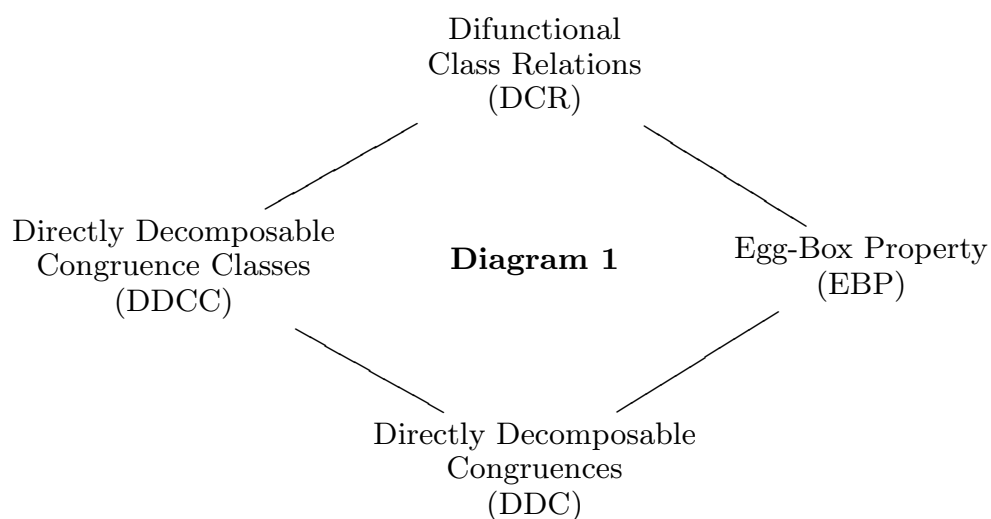

We show at the end of the paper that we can fit congruence-modularity as the top vertex in a similar diamond, whose middle two vertices are congruencedistributivity and congruence-permutability.

It is worth mentioning that Diagram 1 collapses in the case of congruence modular varieties: (DCR) is equivalent to (EBP) simply because every congruence modular variety has (EBP), and (DDCC) is equivalent to (DDC). The last two statements follow from remarks above and they are also found in [5] as Theorems 11.0.17 and 11.0.10, respectively.

To summarize, the interest in the property (DCR) that we study in this paper, is given by the following points: 
- (DCR) is a weakening of congruence permutability, which is implied by congruence-modularity,

- varieties satisfying (DCR) can be characterized similarly to congruence permutable varieties,

- (DCR) is a Mal'tsev condition,

- (DCR) is, in a suitable natural sense explained above, a common generalization of (DDCC) and (EBP).

\section{Characterization theorems for difunctionality of class relations}

In this section we study the property of a variety to have all class relations difunctional. By a class relation, relative to a variety $\mathcal{V}$ (or a $\mathcal{V}$-class relation, in short), we mean a relation $R$ on a set $S$ such that there exists an algebra $A$ in $\mathcal{V}$ for which $S \subseteq A$ and $R$ is the intersection of $S^{2}$ and a class of some congruence $C$ on $A^{2}$. Recall that a binary relation $R$ from a set $X$ to a set $U$ is said to be difunctional, if

$$
x R u \wedge x R v \wedge y R v \Rightarrow y R u
$$

holds for all $x, y \in X$ and $u, v \in U$. Equivalently, $R$ is difunctional when $R R^{\circ} R \subseteq R$ (or when $R R^{\circ} R=R$ ). Recall from [2] that:

- $R$ is difunctional if and only if the relation $Q$ on $R$ defined via the left hand side of the above implication is an equivalence relation:

$$
(x, u) Q(y, v) \Leftrightarrow x R u \wedge x R v \wedge y R v .
$$

- $R$ is difunctional as soon as $Q$ is either symmetric or transitive, and $Q$ is always reflexive.

These facts can be used to establish the well-known characterizations of congruence-permutable varieties using difunctionality of homomorphic relations and reflexivity and symmetry of homomorphic relations, first obtained in [16] and [21], respectively. We would like to adapt these results for class relations in the place of homomorphic relations.

Lemma 1.1 Let $X$ and $U$ be algebras in a variety $\mathcal{V}$, and let $R$ be a congruence class for some congruence $C$ on $X \times U$. Then the relation $Q$ defined above is a $\mathcal{V}$-class relation on $R$.

The relation $Q$ can be obtained as the intersection of $R^{2}$ with the inverse image of this congruence class under the homomorphism $(X \times U)^{2} \rightarrow X \times$ $U$, which maps $((x, u),(y, v))$ to $(x, v)$. Since inverse images of congruence 
classes under homomorphisms are congruence classes, we obtain that $Q$ is a class relation on $R$.

The relation $Q$ can be alternatively constructed as follows. Consider the kernel congruences $\mathrm{Eq}\left(\pi_{1}\right)$ and $\mathrm{Eq}\left(\pi_{2}\right)$ of the product projections

$$
X \ll \stackrel{\pi_{1}}{\longleftarrow} X \times U \stackrel{\pi_{2}}{\longrightarrow} U
$$

Then $Q$ can be obtained as the relational composite

$$
Q=\left(R^{2} \cap \operatorname{Eq}\left(\pi_{1}\right)\right)\left(R^{2} \cap \operatorname{Eq}\left(\pi_{2}\right)\right) .
$$

Note that the order in our notation for a composite of relations is opposite to the usual order used for composites of functions. Writing $r_{1}$ and $r_{2}$ for the product projections restricted to $R$, we have:

Lemma 1.2 $Q=\operatorname{Eq}\left(r_{1}\right) \operatorname{Eq}\left(r_{2}\right)$.

Our first characterization theorem is:

Theorem 1.3 For a variety $\mathcal{V}$ of algebras, the following conditions are equivalent:

(a) Every relation $R \subseteq X \times U$ between algebras $X$ and $U$ in $\mathcal{V}$, such that $R$ is a congruence class, is difunctional.

(b) Every $\mathcal{V}$-class relation $R \subseteq S^{2}$ is difunctional.

(c) Every reflexive $\mathcal{V}$-class relation $R \subseteq S^{2}$ is an equivalence relation.

(d) Every reflexive $\mathcal{V}$-class relation $R \subseteq S^{2}$ is symmetric.

(e) For any two reflexive and symmetric relations $E_{1}$ and $E_{2}$ on a set $S$, we have $E_{1} E_{2}=E_{2} E_{1}$ provided the composite $E_{1} E_{2}$ is a $\mathcal{V}$-class relation.

(f) For any two equivalence relations $E_{1}$ and $E_{2}$ on a set $S$, we have $E_{1} E_{2}=$ $E_{2} E_{1}$ provided the composite $E_{1} E_{2}$ is a $\mathcal{V}$-class relation.

(g) Every reflexive $\mathcal{V}$-class relation $R \subseteq S^{2}$ is transitive.

(a) $\Rightarrow(\mathrm{b})$ : Let $R=S^{2} \cap K$ where $K$ is a congruence class for a congruence $C$ on $A^{2}$, and $A$ is an algebra in $\mathcal{V}$ such that $S \subseteq A$. Now, (a) implies that $K$ is a difunctional relation on $A . S^{2}$ is also trivially a difunctional relation on $A$. The intersection of any two difunctional relations is clearly difunctional, and so, if (a) holds then $R$ is difunctional.

(b) $\Rightarrow(c)$ since every reflexive difunctional relation is an equivalence relation.

$(\mathrm{c}) \Rightarrow(\mathrm{d})$ and $(\mathrm{c}) \Rightarrow(\mathrm{g})$ are trivial.

$(\mathrm{d}) \Rightarrow(\mathrm{e})$ : When $E_{1}$ and $E_{2}$ are reflexive, their composite $E_{1} E_{2}$ is also reflexive. When $E_{1}$ and $E_{2}$ each are symmetric, $E_{2} E_{1}=E_{2}^{\circ} E_{1}^{\circ}=\left(E_{1} E_{2}\right)^{\circ}$. Symmetry of $E_{1} E_{2}$ would further give $E_{2} E_{1}=\left(E_{1} E_{2}\right)^{\circ}=E_{1} E_{2}$.

$(\mathrm{e}) \Rightarrow(\mathrm{f})$ is obvious. 
(f) $\Rightarrow$ (a) follows from Lemmas 1.1 and 1.2, and the fact that $\mathrm{Eq}\left(r_{1}\right) \mathrm{Eq}\left(r_{2}\right)=$ $\mathrm{Eq}\left(r_{2}\right) \mathrm{Eq}\left(r_{1}\right)$ is equivalent to difunctionality of $R$.

$(\mathrm{g}) \Rightarrow$ (a) follows from Lemma 1.1 and the fact that transitivity of $Q$ is equivalent to difunctionality of $R$.

Remark 1.4 The notion of a class relation can be generalized to relations between two different sets. Let $R$ be a relation $R \subseteq S \times T$. Call it a $\mathcal{V}$-class relation when $R=S \times T \cap K$ where $S \subseteq X$ and $T \subseteq U$ for some algebras $X$ and $U$ in the variety $\mathcal{V}$, and $K$ is a class for a congruence $C$ on $X \times U$. Then the conditions in the above theorem are also equivalent to every such relation being difunctional. This condition follows from (a) similarly as does (b) in the theorem, and conversely, it trivially implies both (a) and (b).

Remark 1.5 Notice that the theorem above is in fact valid (without changing the proof) when $\mathcal{V}$ is a class of algebras closed under finite products and subalgebras.

A classical result in universal algebra, obtained in [17], states that a variety is congruence-permutable if and only if the system of term equations

$$
\begin{aligned}
& p(x, x, y)=y, \\
& p(y, x, x)=y,
\end{aligned}
$$

is solvable in the algebraic theory of the variety, i.e., it is possible to express a ternary term $p$ using basic operations of the theory, so that these equations are consequences of the axioms of the algebraic theory.

We can easily get an equational characterization for difunctionality of class relations on algebras in the variety, by employing the standard universal-algebraic technique and characterize when is the congruence $C$ on $\operatorname{Fr}\{x, y\}^{2}$ generated by identifying the elements of the set

$$
\{(x, y),(x, x),(y, x)\}
$$

such that its equivalence class containing these elements also contains the element $(y, y)$. These pairs have been chosen according to the triple $(x, u),(x, v)$, $(y, v)$ appearing in the left hand side of the implication that defines a difunctional relation, by setting $u=y$ and $v=x$. This will not result in loss of generality for getting the Mal'tsev condition. Note that if we have generated a subalgebra of $\operatorname{Fr}\{x, y\}^{2}$ from these pairs, and then impose it to contain $(y, y)$, we would get the equational characterization of congruence permutable varieties recalled above. In our case, we are essentially generating a congruence class containing the three pairs.

What follows is an argument employing a standard technique in the theory of Mal'tsev conditions. First we show that the congruence $C$ above can be obtained by taking the transitive closure of the relation $P_{1}$ on $\operatorname{Fr}\{x, y\}^{2}$ obtained by closing the relation

$$
P_{0}=\{((x, y),(x, x)),((x, x),(y, x)),((y, x),(x, y))\}
$$


under ternary polynomial translations; in other words, elements of $P_{1}$ are ordered pairs $(a, b)$ whose components are

$$
\begin{aligned}
& a=p\left((x, y),(x, x),(y, x),\left(s_{1}(x, y), t_{1}(x, y)\right), \ldots,\left(s_{m}(x, y), t_{m}(x, y)\right)\right) \\
& b=p\left((x, x),(y, x),(x, y),\left(s_{1}(x, y), t_{1}(x, y)\right), \ldots,\left(s_{m}(x, y), t_{m}(x, y)\right)\right)
\end{aligned}
$$

where $p$ is some $(m+3)$-ary term and $s_{i}(x, y), t_{i}(x, y)$ represent arbitrary elements of $\operatorname{Fr}\{x, y\}$ (which are of course determined by some binary terms $s_{i}, t_{i}$ ). Notice that $P_{1}$ is already a homomorphic reflexive relation. In fact, it is the smallest reflexive homomorphic relation on $\operatorname{Fr}\{x, y\}^{2}$ containing $P_{0}$. It is not yet symmetric, however, its transitive closure will be since $P_{1}^{\circ} \subseteq P_{1} P_{1}$. This inclusion itself is a consequence of a similar inclusion for $P_{0}$. Since $P_{1}$ is homomorphic and reflexive, so is its transitive closure. So the transitive closure of $P_{1}$ is indeed the desired congruence.

Theorem 1.6 For a variety $\mathcal{V}$ of algebras, class relations between algebras in it are difunctional if and only if the following system of term equations is solvable in its algebraic theory:

$$
\begin{aligned}
p_{1}\left(x, x, y, s_{1}(x, y), \ldots, s_{m}(x, y)\right) & =x, \\
p_{n}\left(x, y, x, s_{1}(x, y), \ldots, s_{m}(x, y)\right) & =y, \\
p_{1}\left(y, x, x, t_{1}(x, y), \ldots, t_{m}(x, y)\right) & =x, \\
p_{n}\left(x, x, y, t_{1}(x, y), \ldots, t_{m}(x, y)\right) & =y, \\
p_{i}\left(x, y, x, s_{1}(x, y), \ldots, s_{m}(x, y)\right) & =p_{i+1}\left(x, x, y, s_{1}(x, y), \ldots, s_{m}(x, y)\right), \\
p_{i}\left(x, x, y, t_{1}(x, y), \ldots, t_{m}(x, y)\right) & =p_{i+1}\left(y, x, x, t_{1}(x, y), \ldots, t_{m}(x, y)\right), \\
i \in\{1, \ldots, n-1\} . &
\end{aligned}
$$

Let $P_{1}$ be as above. If class relations on algebras in $\mathcal{V}$ are difunctional, then the equivalence classes of the transitive closure of $P_{1}$ must be difunctional, since as we explained above, the transitive closure of $P_{1}$ is a congruence. In particular, this implies that the transitive closure of $P_{1}$ relates $(x, x)$ with $(y, y)$. So there exists a chain

$$
(x, x)=\left(a_{1}, b_{1}\right) P_{1}\left(a_{2}, b_{2}\right) P_{1}\left(a_{3}, b_{3}\right) P_{1} \cdots P_{1}\left(a_{n}, b_{n}\right)=(y, y) .
$$

To get the equations in the theorem, we write out the components of each related pair $\left(\left(a_{i}, b_{i}\right),\left(a_{i+1}, b_{i+1}\right)\right)$ — they are:

$$
\begin{aligned}
& p_{i}\left((x, y),(x, x),(y, x),\left(s_{i 1}(x, y), t_{i 1}(x, y)\right), \ldots,\left(s_{i m_{i}}(x, y), t_{i m_{i}}(x, y)\right)\right), \\
& p_{i}\left((x, x),(y, x),(x, y),\left(s_{i 1}(x, y), t_{i 1}(x, y)\right), \ldots,\left(s_{i m_{i}}(x, y), t_{i m_{i}}(x, y)\right)\right) .
\end{aligned}
$$

It remains to notice that without loss of generality we may assume that $s_{i j}$ 's, $t_{i j}$ 's and $m_{i}$ do not depend on $i$ (each term $p_{i}$ can be expanded by adding superfluous variables so that the above expressions include within it all instances of $\left(s_{i j}, t_{i j}\right)$ in a fixed order). This proves the 'only if' part of the theorem. For the 'if' part, we can use the terms satisfying the equations given in the theorem to show that every class relation is difunctional, by, in some sense, going backwards along the procedure used to obtain these terms. 


\section{Related geometric properties}

Condition (a) in Theorem 1.3 can be expressed by the following diagram:

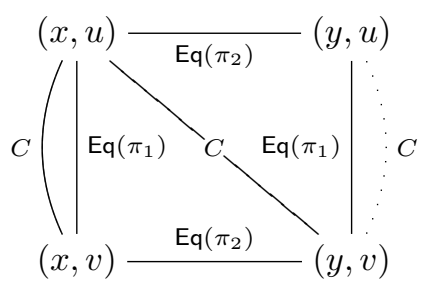

(DCR)

In this diagram,

- $x, y$ are elements of an algebra $X$ and $u, v$ are elements of an algebra $U$,

- $C$ is a congruence on $X \times U$.

The diagram expresses the property that whenever $(x, v) C(x, u) C(y, u)$, also $(y, v) C(y, u)$. This diagram arises by superimposing one of the following diagrams onto the other:

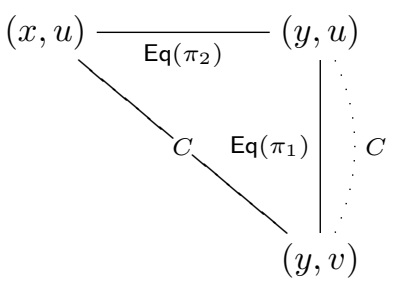

(DDCC)

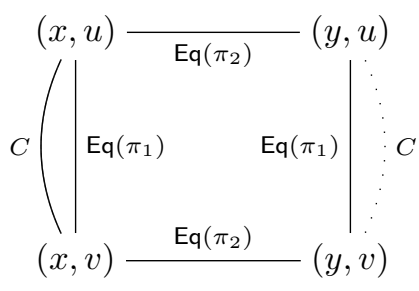

$(\mathrm{EBP})$

Recall from the Introduction that

- the left diagram expresses the property (DDCC);

- the right diagram expresses the property (EBP);

- (DCR) follows both from (DDCC) and from (EBP);

- conjunction of (DDCC) and (EBP) gives the property (DDC).

The counterexample given on page 23 in [6] can be used to confirm that there is a variety having the property (DDCC), but not the property (DDC). This shows that (DDCC) cannot imply (EBP). On the other hand, for the variety of abelian groups for instance, it is easy to see that (EBP) holds, but (DDC) fails. So neither does (EBP) imply (DDCC). We then have a diagram of distinct properties (see Diagram 1 in the Introduction), where the top vertex is obtained from the middle two vertices by superimposition, and the bottom vertex is the 
conjunction of the middle vertices. Moreover, the bottom vertex may also be obtained through a combination of the diagrams for (DDCC) and (EBP) as we now explain. Recall from [11] that a variety has (DDC) if and only if congruences on cartesian products satisfy

$$
(x, u) C(y, v) \quad \Rightarrow \quad(z, u) C(z, v)
$$

for all $x, y, z, u, v$. We can thus express this property using the diagram

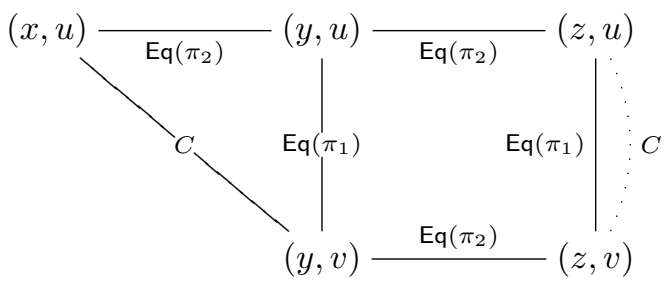

(DDC)

which can be obtained by adjoining the diagrams for (DDCC) and (EBP) sideby-side, where the dotted edge of the left diagram cancels the matching solid edge of the right diagram.

There is a natural way of strengthening the four properties considered above by requiring $C$ to be a congruence defined on a subalgebra $A$ of $X \times U$ containing all vertices in the diagram. The following theorem is likely to be folklore, although we could not find it in the literature.

Theorem 2.1 In a variety of algebras the shifting lemma holds if and only if the property

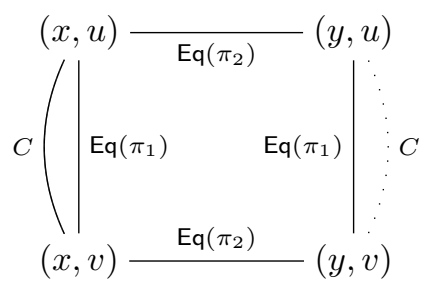

holds for those pairs $(x, u),(x, v),(y, u),(y, v)$ which belong to a subalgebra $A$ of the product $X \times U$ and where $C$ is a congruence on $A$.

Since the vertices of the diagram given in the theorem are required to be elements of $A$, we can think of this diagram as a diagram in $A$. Writing $R$ and $S$ for the congruences $R=A^{2} \cap \mathrm{Eq}\left(\pi_{1}\right)$ and $S=A^{2} \cap \mathrm{Eq}\left(\pi_{2}\right)$, we get:

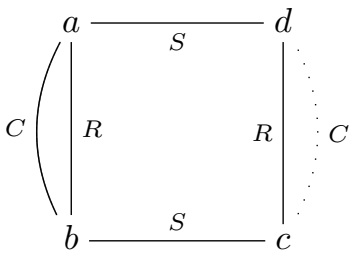


Note that $R \cap S$ is the smallest congruence on $A$, and so trivially $R \cap S \subseteq C$. Thus, this is an evident consequence of the shifting lemma. Moreover, the shifting lemma can be reduced to this, since when we have the above diagram with $R \cap S \subseteq C$, we could project it on $A^{\prime}=A /(R \cap S)$ in which $R \cap S$ becomes the smallest congruence, and so $A^{\prime}$ can be preseted as a subalgebra of $A / R \times A / S$. This argument will use the fact that the image of $C$ under the projection $A \rightarrow A /(R \cap S)$ is still a congruence, which is a consequence of the inclusion $R \cap S \subseteq C$.

The theorem above links up the diagram for (EBP) and the diagram for the shifting lemma. There are similar links between the diagram for (DDCC) and the diagram for the triangular lemma in the sense of [10], as well as the diagram for (DDC) (after removing the superflous vertex $(y, u)$ ) and the diagram for the upright principle in the sense of [9] (which in [4] is called the trapezoid lemma). This latter property is equivalent to congruence-distributivity ([9], [4]). A similar modification to the diagram for (DCR) will result in the property

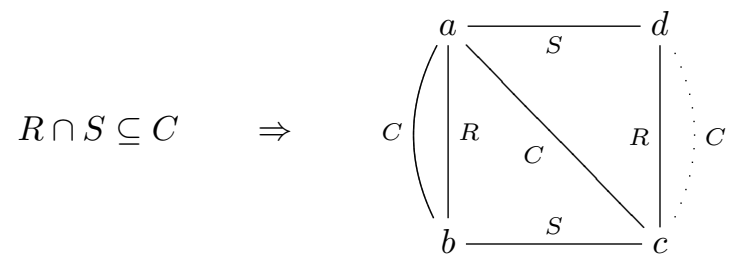

that does not seem to appear in the literature; we leave its investigation for future work. We only note here that this property is also a Mal'tsev condition given by the following scheme:

$$
\begin{cases}p_{0}(x, u, v, y)=x & \\ p_{n}(x, u, v, y)=y & \\ p_{i}(x, y, y, x)=p_{i+1}(x, y, y, x), & \text { for } i \text { even } \\ p_{i}(x, x, y, y)=p_{i+1}(x, x, y, y), & \text { for } i \text { even, } \\ p_{i}(x, x, x, y)=p_{i+1}(x, x, x, y), & \text { for } i \text { odd. }\end{cases}
$$

Replacing the last line as follows, the system of equations becomes equivalent to the one for the quaternary terms given in [7] characterizing congruencemodularity:

$$
\left\{\begin{array}{l}
p_{0}(x, u, v, y)=x \\
p_{n}(x, u, v, y)=y \\
p_{i}(x, y, y, x)=p_{i+1}(x, y, y, x), \quad \text { for } i \text { even, } \\
p_{i}(x, x, y, y)=p_{i+1}(x, x, y, y), \quad \text { for } i \text { even, } \\
p_{i}(x, u, u, y)=p_{i+1}(x, u, u, y), \quad \text { for } i \text { odd }
\end{array}\right.
$$

To get the equations for the Day terms, we just need to observe that thanks to the first and the last lines, the third line above can be replaced with $p_{i}(x, y, y, x)=$ $x$ (for all $i$ ). This connection with congruence-modularity can be explained by noting that the diagram for our property is the same as the diagram for the 
shifting lemma with additional diagonal $C$-line inserted. Similarly, the first scheme above is closely related to the one given in [6] characterizing varieties satisfying the triangular lemma.

\section{Counterexamples}

The counterexample given in [13] on page 66 can be used to show that the property identified at the end of the last section is strictly stronger than (DCR). This counterexample is the variety of algebras given by a ternary operation $p$ and a constant 0 satisfying $p(x, x, y)=y$ and $p(y, 0,0)=y$. This variety is a Jónsson-Tarski variety [15] and at the same time a subtractive variety in the sense of [20] (see also [14]). As we will now show, in general neither of these two types of varieties have (DCR).

Theorem 3.1 Not every subtractive variety has difunctional class relations.

Consider the variety of subtraction algebras, i.e., algebraic structures with one constant 0 and one binary operation $s$ satisfying $s(x, x)=0$ and $s(x, 0)=x$. In this variety, consider the algebra whose underlying set is $X=\{0,1,2\}$ and where $s$ is defined by

$$
s(x, y)= \begin{cases}x, & y=0 \\ 0, & y \neq 0\end{cases}
$$

The same type of subtraction was used in [1] to give a counterexample to congruence-modularity of subtraction algebras. The operation on the product $X \times X$ for such $X$ is given by the following table (for brevity, we write an ordered pair $(a, b)$ as $a b$, and ' $*$ ' for the pair $00=(0,0))$ :

\begin{tabular}{c|c|cc|cc|c|ccc}
$s$ & $*$ & 01 & 02 & 10 & 20 & 11 & 12 & 21 & 22 \\
\hline$*$ & $*$ & $*$ & $*$ & $*$ & $*$ & $*$ & $*$ & $*$ & $*$ \\
\hline 01 & 01 & $*$ & $*$ & 01 & 01 & $*$ & $*$ & $*$ & $*$ \\
02 & 02 & $*$ & $*$ & 02 & 02 & $*$ & $*$ & $*$ & $*$ \\
\hline 10 & 10 & 10 & 10 & $*$ & $*$ & $*$ & $*$ & $*$ & $*$ \\
20 & 20 & 20 & 20 & $*$ & $*$ & $*$ & $*$ & $*$ & $*$ \\
\hline 11 & 11 & 10 & 10 & 01 & 01 & $*$ & $*$ & $*$ & $*$ \\
\hline 12 & 12 & 10 & 10 & 02 & 02 & $*$ & $*$ & $*$ & $*$ \\
21 & 21 & 20 & 20 & 01 & 01 & $*$ & $*$ & $*$ & $*$ \\
22 & 22 & 20 & 20 & 02 & 02 & $*$ & $*$ & $*$ & $*$
\end{tabular}

From this table we can see that the equivalence relation on $X \times X$ with equivalence classes given by

$$
\{*\}, \quad\{01,02\}, \quad\{10,20\}, \quad\{11\}, \quad\{12,21,22\}
$$

is a congruence. The relation $\{12,21,22\}$ is thus a class relation on $X$, but it is not difunctional since it includes 21, 22 and 12, but not 11 . 
Theorem 3.2 Not every Jónsson-Tarski variety has difunctional class relations.

In the variety of semilattices (or alternatively, of idempotent commutative monoids), consider the underlying structure $X=(\{0,1\}, \vee)$ of the two-element Boolean algebra. The operation on the product $X \times X$ is then given by the following table:

\begin{tabular}{c|c|ccc}
$\vee$ & 00 & 01 & 10 & 11 \\
\hline 00 & 00 & 01 & 10 & 11 \\
\hline 01 & 01 & 01 & 11 & 11 \\
10 & 10 & 11 & 10 & 11 \\
11 & 11 & 11 & 11 & 11
\end{tabular}

From this table we can see that the equivalence relation on $X \times X$ with equivalence classes given by

$$
\{10,11,01\}, \quad\{00\}
$$

is a congruence. The relation $\{10,11,01\}$ is thus a class relation on $X$, but it is not difunctional.

\section{Congruence-modularity is permutability su- perimposed on distributivity}

We conclude with a remark that the following diamond is actually of a similar type as Diagram 1 in the Introduction:

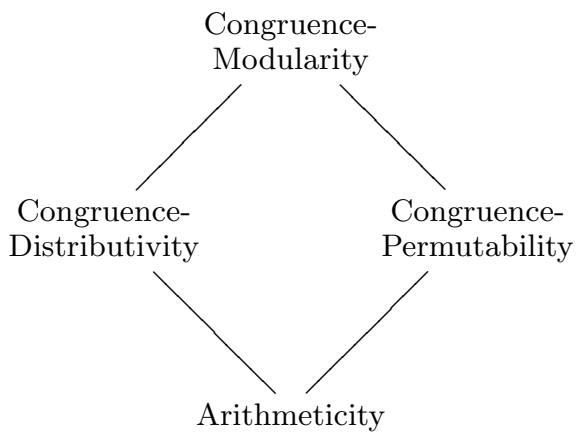

The trapezoid lemma which characterizes congruence-distributivity can be equivalently rephrased as the following geometric property:

$$
\left.R \cap S \subseteq C \quad \Rightarrow \quad C\right|_{b} ^{a-\left.d \bar{S}\right|_{S} ^{S} e}{ }_{c}{ }_{C}
$$


At the same time, congruence permutability can be represented by a property with the dotted part matching the one in the previous diagram:

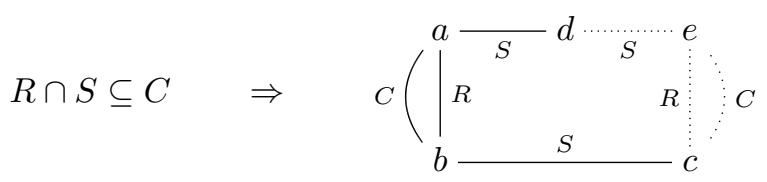

Superimposing the second diagram onto the first one gives a property that is easily seen to be equivalent to the shifting lemma:

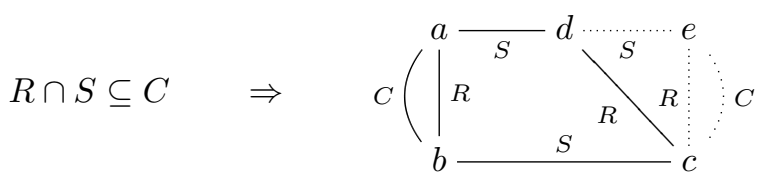

On the other hand, adjoining them we obtain the property

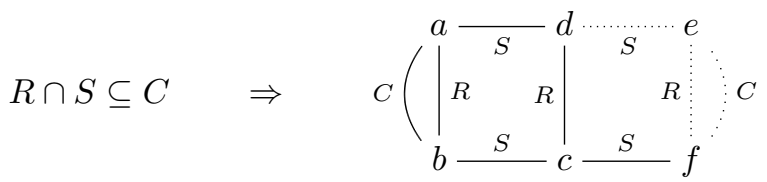

that is easily seen to be equivalent to the conjunction of congruence-distributivity and congruence-permutability, and hence, it holds if and only if the variety is arithmetical (see [18]).

The fact that congruence-modularity has a geometric presentation which is obtained by superimposing congruence-permutability onto congruence-distributivity gives an alternative interpretation to the slogan 'congruence-modularity is permutability composed with distributivity' from [12].

Acknowledgements. We would like to thank the anonymous referees as well as Hans-Peter Gumm, for their useful remarks on earlier versions of this paper.

\section{References}

[1] Agliano, P., Ursini, A.: Ideals and other generalizations of congruence classes. J. Aust. Math. Soc. 53, 103-115 (1992)

[2] Carboni, A., Pedicchio, M.C., Pirovano, N.: Internal graphs and internal groupoids in Mal'tsev categories. Canadian Math. Soc. Conference proceedings 13, 97-109 (1992)

[3] Chajda, I.: The egg-box property of congruences. Math. Slovaca 38, 243247 (1988) 
[4] Chajda, I., Czédli, G., Horvaáth, E.K.: Trapezoid lemma and congruence distributivity. Math. Slovaca 53, 247-253 (2003)

[5] Chajda, I., Eigenthaler, G., Länger, H.: Congruence Classes in Universal Algebra. Research Expositions in Mathematics 26, Heldermann Verlag (2003)

[6] Chajda, I., Halas, R.: Varieties satisfying the triangular scheme need not be conguence distributive. Acta Univ. Palack. Olomuc. Fac. Rerum Natur. Math. 46, 19-24 (2007)

[7] Day, A.: A characterization of modularity for congruence lattices of algebras. Canad. Math. Bull. 12, 167-173 (1969)

[8] Duda, J.: Varieties having directly decomposable congruence classes. Časopis pro pěstování matematiky 111, 394-403 (1986)

[9] Duda, J.: The upright principle for congruence distributive varieties. Abstract of a lecture seminar presented in Brno, March (2000)

[10] Duda, J.: The triangular principle for congruence distributive varieties. Abstract of a lecture seminar presented in Brno, March (2000)

[11] Fraser, G.A., Horn, A.: Congruence relations in direct products. Proc. Amer. Math. Soc. 26, 390-394 (1970)

[12] Gumm, H.P.: Congruence modularity is permutability composed with distributivity. Arch. Math. (Basel) 36, 569-576 (1981)

[13] Gumm, H.P.: Geometrical methods in congruence modular algebras. Mem. Amer. Math. Soc. 45 (1983)

[14] Gumm, H.P., Ursini, A.: Ideals in universal algebra. Algebra Universalis 19, 45-54 (1984)

[15] Jónsson, B., Tarski, A.: Direct Decompositions of Finite Algebraic Systems. Notre Dame Mathematical Lectures 5, University of Notre Dame, Indiana (1947)

[16] Lambek, J.: Goursat's theorem and the Zassenhaus lemma. Canad. J. Math. 10, 45-56 (1957)

[17] Mal'tsev, A.I.: On the general theory of algebraic systems. Mat. Sbornik, N.S. 35, 3-20 (1954) (Russian)

[18] Pixley, A.F.: Distributivity and permutability in equational classes. Proc. Amer. Math. Soc. 14, 105-109 (1963)

[19] Riguet, J.: Relations binaires, fermetures, correspondances de Galois. Bull. Soc. Math. France 76, 114-155 (1948) 
[20] Ursini, A.: On subtractive varieties, I. Algebra Universalis 31, 204-222 (1994)

[21] Werner, H.: A Mal'cev condition for admissible relations. Algebra Universalis 3, 263 (1973) 